\title{
Operations of Points on Elliptic Curve in Projective Coordinates
}

\author{
Yuichi Futa \\ Shinshu University \\ Nagano, Japan
}

\author{
Hiroyuki Okazaki ${ }^{1}$ \\ Shinshu University \\ Nagano, Japan \\ Yasunari Shidama ${ }^{2}$ \\ Shinshu University \\ Nagano, Japan
}

\author{
Daichi Mizushima \\ Shinshu University \\ Nagano, Japan
}

Summary. In this article, we formalize operations of points on an elliptic curve over $\mathbf{G F}(\mathbf{p})$. Elliptic curve cryptography [7], whose security is based on a difficulty of discrete logarithm problem of elliptic curves, is important for information security. We prove that the two operations of points: compell ${ }_{\text {ProjCo }}$ and addel $l_{\text {ProjCo }}$ are unary and binary operations of a point over the elliptic curve.

MML identifier: $\underline{\text { EC_PF_2 }}$, version: $\underline{7.12 .02 \quad 4.176 .1140}$

The terminology and notation used here are introduced in the following papers: $[5],[17],[3],[1],[13],[4],[2],[12],[14],[10],[9],[16],[15],[8],[11]$, and [6].

\section{Arithmetic in $\mathbf{G F}(\mathbf{p})$}

For simplicity, we adopt the following convention: $i, j$ denote integers, $n$ denotes a natural number, $K$ denotes a field, and $a_{1}, a_{2}, a_{3}, a_{4}, a_{5}, a_{6}$ denote elements of $K$.

One can prove the following propositions:

(1) If $a_{1}=-a_{2}$, then $a_{1}{ }^{2}=a_{2}{ }^{2}$.

(2) $\left(1_{K}\right)^{-1}=1_{K}$.

\footnotetext{
${ }^{1}$ This work was supported by JSPS KAKENHI 21240001.

${ }^{2}$ This work was supported by JSPS KAKENHI 22300285.
}

(C) 2012 University of Białystok CC-BY-SA License ver. 3.0 or later ISSN 1426-2630(p), 1898-9934(e) 
(3) If $a_{2} \neq 0_{K}$ and $a_{4} \neq 0_{K}$ and $a_{1} \cdot a_{2}{ }^{-1}=a_{3} \cdot a_{4}{ }^{-1}$, then $a_{1} \cdot a_{4}=a_{2} \cdot a_{3}$.

(4) If $a_{2} \neq 0_{K}$ and $a_{4} \neq 0_{K}$ and $a_{1} \cdot a_{4}=a_{2} \cdot a_{3}$, then $a_{1} \cdot a_{2}{ }^{-1}=a_{3} \cdot a_{4}{ }^{-1}$.

(5) If $a_{1}=0_{K}$ and $n>1$, then $a_{1}{ }^{n}=0_{K}$.

(6) If $a_{1}=-a_{2}$, then $-a_{1}=a_{2}$.

(7) $a_{1}+a_{2}+a_{3}+a_{4}=a_{4}+a_{2}+a_{3}+a_{1}$ and $a_{1}+a_{2}+a_{3}+a_{4}=a_{1}+a_{4}+a_{3}+a_{2}$.

(8) $\left(a_{1}+a_{2}+a_{3}\right)+a_{4}=a_{1}+\left(a_{2}+a_{3}+a_{4}\right)$ and $\left(a_{1}+a_{2}+a_{3}+a_{4}\right)+a_{5}=$ $a_{1}+\left(a_{2}+a_{3}+a_{4}+a_{5}\right)$.

(9) $\left(a_{1}+a_{2}+a_{3}+a_{4}+a_{5}\right)+a_{6}=a_{1}+\left(a_{2}+a_{3}+a_{4}+a_{5}+a_{6}\right)$.

(10) $a_{1} \cdot a_{2} \cdot a_{3} \cdot a_{4}=a_{4} \cdot a_{2} \cdot a_{3} \cdot a_{1}$ and $a_{1} \cdot a_{2} \cdot a_{3} \cdot a_{4}=a_{1} \cdot a_{4} \cdot a_{3} \cdot a_{2}$.

(11) $\left(a_{1} \cdot a_{2} \cdot a_{3}\right) \cdot a_{4}=a_{1} \cdot\left(a_{2} \cdot a_{3} \cdot a_{4}\right)$ and $\left(a_{1} \cdot a_{2} \cdot a_{3} \cdot a_{4}\right) \cdot a_{5}=a_{1} \cdot\left(a_{2} \cdot a_{3} \cdot a_{4} \cdot a_{5}\right)$.

(12) $\left(a_{1} \cdot a_{2} \cdot a_{3} \cdot a_{4} \cdot a_{5}\right) \cdot a_{6}=a_{1} \cdot\left(a_{2} \cdot a_{3} \cdot a_{4} \cdot a_{5} \cdot a_{6}\right)$ and $a_{1} \cdot a_{2} \cdot a_{3} \cdot a_{4} \cdot a_{5} \cdot a_{6}=$ $a_{1} \cdot\left(a_{2} \cdot a_{3} \cdot a_{4}\right) \cdot a_{5} \cdot a_{6}$.

(13) $\left(a_{1} \cdot a_{2} \cdot a_{3}\right)^{n}=a_{1}{ }^{n} \cdot a_{2}{ }^{n} \cdot a_{3}{ }^{n}$.

(14) $a_{1} \cdot\left(a_{2}+a_{3}+a_{4}\right)=a_{1} \cdot a_{2}+a_{1} \cdot a_{3}+a_{1} \cdot a_{4}$ and $a_{1} \cdot\left(\left(a_{2}+a_{3}\right)-a_{4}\right)=$ $\left(a_{1} \cdot a_{2}+a_{1} \cdot a_{3}\right)-a_{1} \cdot a_{4}$ and $a_{1} \cdot\left(\left(a_{2}-a_{3}\right)+a_{4}\right)=\left(a_{1} \cdot a_{2}-a_{1} \cdot a_{3}\right)+a_{1} \cdot a_{4}$ and $a_{1} \cdot\left(a_{2}-a_{3}-a_{4}\right)=a_{1} \cdot a_{2}-a_{1} \cdot a_{3}-a_{1} \cdot a_{4}$ and $a_{1} \cdot\left(-a_{2}+a_{3}+a_{4}\right)=$ $-a_{1} \cdot a_{2}+a_{1} \cdot a_{3}+a_{1} \cdot a_{4}$ and $a_{1} \cdot\left(\left(-a_{2}+a_{3}\right)-a_{4}\right)=\left(-a_{1} \cdot a_{2}+a_{1} \cdot a_{3}\right)-a_{1} \cdot a_{4}$ and $a_{1} \cdot\left(\left(-a_{2}-a_{3}\right)+a_{4}\right)=\left(-a_{1} \cdot a_{2}-a_{1} \cdot a_{3}\right)+a_{1} \cdot a_{4}$ and $a_{1} \cdot\left(-a_{2}-a_{3}-a_{4}\right)=$ $-a_{1} \cdot a_{2}-a_{1} \cdot a_{3}-a_{1} \cdot a_{4}$.

(15) $\left(a_{1}+a_{2}\right) \cdot\left(a_{1}-a_{2}\right)=a_{1}^{2}-a_{2}^{2}$.

(16) $\left(a_{1}+a_{2}\right) \cdot\left(\left(a_{1}^{2}-a_{1} \cdot a_{2}\right)+a_{2}^{2}\right)=a_{1}^{3}+a_{2}^{3}$.

(17) $\left(a_{1}-a_{2}\right) \cdot\left(a_{1}^{2}+a_{1} \cdot a_{2}+a_{2}^{2}\right)=a_{1}{ }^{3}-a_{2}{ }^{3}$.

Let $n, p$ be natural numbers. We say that $p$ is $n$ or greater if and only if:

(Def. 1) $n \leq p$.

Let us note that there exists a natural number which is 5 or greater and prime.

The following propositions are true:

(18) For all elements $g_{1}, g_{2}, g_{3}, a$ of $\operatorname{GF}(p)$ such that $g_{1}=i \bmod p$ and $g_{2}=j \bmod p$ and $g_{3}=(i+j) \bmod p$ holds $g_{1} \cdot a+g_{2} \cdot a=g_{3} \cdot a$.

(19) For all elements $g_{1}, g_{2}, a$ of $\operatorname{GF}(p)$ such that $g_{1}=i \bmod p$ and $g_{2}=$ $j$ mod $p$ and $j=i+1$ holds $g_{1} \cdot a+a=g_{2} \cdot a$.

(20) For all elements $g_{4}, a$ of $\operatorname{GF}(p)$ such that $g_{4}=2 \bmod p$ holds $a+a=g_{4} \cdot a$.

(21) For all elements $g_{1}, g_{2}, g_{3}, a$ of $\operatorname{GF}(p)$ such that $g_{1}=i \bmod p$ and $g_{2}=j \bmod p$ and $g_{3}=(i-j) \bmod p$ holds $g_{1} \cdot a-g_{2} \cdot a=g_{3} \cdot a$.

(22) For all elements $g_{1}, g_{2}, a$ of $\mathrm{GF}(p)$ such that $g_{1}=i \bmod p$ and $g_{2}=$ $j \bmod p$ and $i=j+1$ holds $g_{1} \cdot a-g_{2} \cdot a=a$.

(23) For all elements $g_{1}, g_{2}, a$ of $\mathrm{GF}(p)$ such that $g_{1}=i \bmod p$ and $g_{2}=$ $j \bmod p$ and $i=j+1$ holds $g_{1} \cdot a-a=g_{2} \cdot a$. 
(24) For all elements $g_{4}, a$ of $\mathrm{GF}(p)$ such that $g_{4}=2 \bmod p$ holds $g_{4} \cdot a-a=a$.

(25) For all elements $g_{4}, a, b$ of $\operatorname{GF}(p)$ such that $g_{4}=2 \bmod p$ holds $(a+b)^{2}=$ $a^{2}+g_{4} \cdot a \cdot b+b^{2}$.

(26) For all elements $g_{4}, a, b$ of $\operatorname{GF}(p)$ such that $g_{4}=2 \bmod p$ holds $(a-b)^{2}=$ $\left(a^{2}-g_{4} \cdot a \cdot b\right)+b^{2}$.

(27) For all elements $g_{4}, a, b, c, d$ of $\mathrm{GF}(p)$ such that $g_{4}=2 \bmod p$ holds $(a \cdot c+b \cdot d)^{2}=a^{2} \cdot c^{2}+g_{4} \cdot a \cdot b \cdot c \cdot d+b^{2} \cdot d^{2}$.

(28) Let $p$ be a prime number, $n$ be a natural number, and $g_{4}$ be an element of $\mathrm{GF}(p)$. If $p>2$ and $g_{4}=2 \bmod p$, then $g_{4} \neq 0_{\mathrm{GF}(p)}$ and $g_{4}{ }^{n} \neq 0_{\mathrm{GF}(p)}$.

(29) Let $p$ be a prime number, $n$ be a natural number, and $g_{4}, g_{5}$ be elements of $\mathrm{GF}(p)$. If $p>3$ and $g_{5}=3 \bmod p$, then $g_{5} \neq 0_{\mathrm{GF}(p)}$ and $g_{5}{ }^{n} \neq 0_{\mathrm{GF}(p)}$.

\section{Parameters of an Elliptic Curve}

Let $p$ be a 5 or greater prime number. The parameters of elliptic curve $p$ yielding a subset of (the carrier of $\mathrm{GF}(p)) \times($ the carrier of $\mathrm{GF}(p))$ is defined as follows:

(Def. 2) The parameters of elliptic curve $p=\{\langle a, b\rangle$; $a$ ranges over elements of $\mathrm{GF}(p), b$ ranges over elements of $\operatorname{GF}(p)$ : $\left.\operatorname{Disc}(a) \neq 0_{\mathrm{GF}(p)}\right\}$.

Let $p$ be a 5 or greater prime number. Observe that the parameters of elliptic curve $p$ is non empty.

Let $p$ be a 5 or greater prime number and let $z$ be an element of the parameters of elliptic curve $p$. Then $z_{\mathbf{1}}$ is an element of $\operatorname{GF}(p)$. Then $z_{\mathbf{2}}$ is an element of $\operatorname{GF}(p)$.

The following proposition is true

(30) Let $p$ be a 5 or greater prime number and $z$ be an element of the parameters of elliptic curve $p$. Then $p>3$ and $\operatorname{Disc}\left(z_{1}\right) \neq 0_{\mathrm{GF}(p)}$.

For simplicity, we adopt the following rules: $p_{1}, p_{2}, p_{3}$ denote sets, $P_{1}, P_{2}$, $P_{3}$ denote elements of $\mathrm{GF}(p), P$ denotes an element of $\operatorname{ProjCo}(\mathrm{GF}(p))$, and $O$ denotes an element of $\mathrm{EC}_{\mathrm{SetProjCo}}(a)$.

Let $p$ be a prime number, let $a, b$ be elements of $\operatorname{GF}(p)$, and let $P$ be an element of $\operatorname{EC}_{\text {SetProjCo }}(a)$. The functor $P_{\mathbf{1}}$ yields an element of $\mathrm{GF}(p)$ and is defined as follows:

(Def. 3) If $P=\left\langle p_{1}, p_{2}, p_{3}\right\rangle$, then $P_{1}=p_{1}$.

The functor $P_{\mathbf{2}}$ yielding an element of $\operatorname{GF}(p)$ is defined as follows:

(Def. 4) If $P=\left\langle p_{1}, p_{2}, p_{3}\right\rangle$, then $P_{2}=p_{2}$.

The functor $P_{\mathbf{3}}$ yielding an element of $\mathrm{GF}(p)$ is defined by:

(Def. 5) If $P=\left\langle p_{1}, p_{2}, p_{3}\right\rangle$, then $P_{3}=p_{3}$.

We now state three propositions: 
(31) For every prime number $p$ and for all elements $a, b$ of $\operatorname{GF}(p)$ and for every element $P$ of $\mathrm{EC}_{\text {SetProjCo }}(a)$ holds $P=\left\langle P_{\mathbf{1}}, P_{\mathbf{2}}, P_{\mathbf{3}}\right\rangle$.

(32) Let $p$ be a prime number, $a, b$ be elements of $\operatorname{GF}(p), P$ be an element of $\operatorname{EC}_{\text {SetProjCo }}(a)$, and $Q$ be an element of $\operatorname{ProjCo}(\mathrm{GF}(p))$. Then $P=Q$ if and only if the following conditions are satisfied:

(i) $P_{1}=Q_{1}$,

(ii) $P_{2}=Q_{2}$, and

(iii) $P_{\mathbf{3}}=Q_{\mathbf{3}}$.

(33) Let $p$ be a prime number, $a, b, P_{1}, P_{2}, P_{3}$ be elements of $\mathrm{GF}(p)$, and $P$ be an element of $\mathrm{EC}_{\mathrm{SetProjCo}}(a)$. If $P=\left\langle P_{1}, P_{2}, P_{3}\right\rangle$, then $P_{1}=P_{1}$ and $P_{2}=P_{2}$ and $P_{3}=P_{3}$.

Let $p$ be a prime number, let $P$ be an element of $\operatorname{ProjCo}(\mathrm{GF}(p))$, and let $C_{1}$ be a function from (the carrier of $\operatorname{GF}(p)) \times($ the carrier of $\operatorname{GF}(p)) \times$ (the carrier of $\operatorname{GF}(p))$ into $\operatorname{GF}(p)$. We say that $P$ is on curve defined by an equation $C_{1}$ if and only if:

(Def. 6) $\quad C_{1}(P)=0_{\mathrm{GF}(p)}$.

The following two propositions are true:

(34) $P$ is on curve defined by an equation $\operatorname{EC}_{\mathrm{WEqProjCo}}(a)$ iff $P$ is an element of $\mathrm{EC}_{\mathrm{SetProjCo}}(a)$.

(35) Let $p$ be a prime number, $a, b$ be elements of $\mathrm{GF}(p)$, and $P$ be an element of $\mathrm{EC}_{\mathrm{SetProjCo}}(a)$. Then $\left(P_{\mathbf{2}}\right)^{2} \cdot P_{\mathbf{3}}-\left(\left(P_{\mathbf{1}}\right)^{3}+a \cdot P_{\mathbf{1}} \cdot\left(P_{\mathbf{3}}\right)^{2}+b \cdot\left(P_{\mathbf{3}}\right)^{3}\right)=0_{\mathrm{GF}(p)}$.

Let $p$ be a prime number and let $P$ be an element of $\operatorname{Proj} \operatorname{Co}(\operatorname{GF}(p))$. The represent point of $P$ yields an element of $\operatorname{ProjCo}(\mathrm{GF}(p))$ and is defined by:

(Def. 7)(i) The represent point of $P=\left\langle P_{\mathbf{1}} \cdot\left(P_{\mathbf{3}}\right)^{-1}, P_{\mathbf{2}} \cdot\left(P_{\mathbf{3}}\right)^{-1}, 1\right\rangle$ if $P_{\mathbf{3}} \neq 0$,

(ii) the represent point of $P=\langle 0,1,0\rangle$ if $P_{\mathbf{3}}=0$,

(iii) $P_{\mathbf{3}}=0$, otherwise.

The following propositions are true:

(36) Let $p$ be a 5 or greater prime number, $z$ be an element of the parameters of elliptic curve $p$, and $P$ be an element of $\operatorname{EC}_{\text {SetProjCo }}\left(z_{1}\right)$. Then the represent point of $P \equiv P$ and the represent point of $P \in \operatorname{EC}_{\mathrm{SetProjCo}}\left(z_{1}\right)$.

(37) Let $p$ be a prime number, $a, b$ be elements of $\mathrm{GF}(p)$, and $P$ be an element of $\operatorname{Proj} \mathrm{Co}(\mathrm{GF}(p))$. Suppose (the represent point of $P)_{\mathbf{3}}=0$. Then the represent point of $P=\langle 0,1,0\rangle$ and $P_{\mathbf{3}}=0$.

(38) Let $p$ be a prime number, $a, b$ be elements of $\mathrm{GF}(p)$, and $P$ be an element of $\operatorname{Proj} \mathrm{Co}(\mathrm{GF}(p))$. Suppose (the represent point of $P)_{\mathbf{3}} \neq 0$. Then the represent point of $P=\left\langle P_{\mathbf{1}} \cdot\left(P_{\mathbf{3}}\right)^{-1}, P_{\mathbf{2}} \cdot\left(P_{\mathbf{3}}\right)^{-1}, 1\right\rangle$ and $P_{\mathbf{3}} \neq 0$.

(39) Let $p$ be a 5 or greater prime number, $z$ be an element of the parameters of elliptic curve $p$, and $P, Q$ be elements of $\operatorname{EC}_{\text {SetProjCo }}\left(z_{1}\right)$. Then $P \equiv Q$ if and only if the represent point of $P=$ the represent point of $Q$. 


\section{Operations of Points on an Elliptic Curve over GF(p)}

Let $p$ be a 5 or greater prime number and let $z$ be an element of the parameters of elliptic curve $p$. The functor compell $\operatorname{ProjCo}_{2}(z, p)$ yields a function from $\mathrm{EC}_{\mathrm{SetProjCo}}\left(z_{1}\right)$ into $\mathrm{EC}_{\text {SetProjCo }}\left(z_{1}\right)$ and is defined as follows:

(Def. 8) For every element $P$ of $\operatorname{EC}_{\text {SetProjCo }}\left(z_{1}\right)$ holds $\left(\operatorname{compell}_{\text {ProjCo }}(z, p)\right)(P)=$ $\left\langle P_{1},-P_{2}, P_{3}\right\rangle$.

Let $p$ be a 5 or greater prime number, let $z$ be an element of the parameters of elliptic curve $p$, let $F$ be a function from $\operatorname{EC}_{\text {SetProjCo }}\left(z_{1}\right)$ into $\operatorname{EC}_{\text {SetProjCo }}\left(z_{1}\right)$, and let $P$ be an element of $\operatorname{EC}_{\text {SetProjCo }}\left(z_{1}\right)$. Then $F(P)$ is an element of $\mathrm{EC}_{\mathrm{SetProjCo}}\left(z_{1}\right)$.

We now state a number of propositions:

(40) Let $p$ be a 5 or greater prime number, $z$ be an element of the parameters of elliptic curve $p$, and $O$ be an element of $\operatorname{EC}_{\mathrm{SetProjCo}}\left(z_{1}\right)$. If $O=\langle 0,1$, $0\rangle$, then $\left(\right.$ compell $\left._{\text {ProjCo }}(z, p)\right)(O) \equiv O$.

(41) Let $p$ be a 5 or greater prime number, $z$ be an element of the parameters of elliptic curve $p$, and $P$ be an element of $\operatorname{EC}_{\text {SetProjCo }}\left(z_{1}\right)$. Then $\left(\operatorname{compell}_{\text {ProjCo }}(z, p)\right)\left(\left(\operatorname{compell}_{\text {ProjCo }}(z, p)\right)(P)\right)=P$.

(42) Let $p$ be a 5 or greater prime number, $z$ be an element of the parameters of elliptic curve $p$, and $P$ be an element of $\operatorname{EC}_{\text {SetProjCo }}\left(z_{1}\right)$. Suppose $P_{\mathbf{3}} \neq 0$. Then the represent point of $\left(\operatorname{compell}_{\mathrm{ProjCo}}(z, p)\right)(P)=$ $\left(\right.$ compell $\left._{\mathrm{ProjCo}}(z, p)\right)$ (the represent point of $P$ ).

(43) Let $p$ be a 5 or greater prime number, $z$ be an element of the parameters of elliptic curve $p$, and $P, Q$ be elements of $\operatorname{EC}_{\text {SetProjCo }}\left(z_{1}\right)$. Then $P=Q$ if and only if $\left(\operatorname{compell}_{\mathrm{ProjCo}}(z, p)\right)(P)=\left(\operatorname{compell}_{\mathrm{ProjCo}}(z, p)\right)(Q)$.

(44) Let $p$ be a 5 or greater prime number, $z$ be an element of the parameters of elliptic curve $p$, and $P$ be an element of $\operatorname{EC}_{\text {SetProjCo }}\left(z_{1}\right)$. If $P_{3} \neq 0$, then $P \equiv\left(\right.$ compell $\left._{\text {ProjCo }}(z, p)\right)(P)$ iff $P_{\mathbf{2}}=0$.

(45) Let $p$ be a 5 or greater prime number, $z$ be an element of the parameters of elliptic curve $p$, and $P, Q$ be elements of $\operatorname{EC}_{\text {SetProjCo }}\left(z_{1}\right)$. If $P_{\mathbf{3}} \neq 0$, then $P_{\mathbf{1}}=Q_{\mathbf{1}}$ and $P_{\mathbf{3}}=Q_{\mathbf{3}}$ iff $P=Q$ or $P=\left(\operatorname{compell}_{\mathrm{ProjCo}}(z, p)\right)(Q)$.

(46) Let $p$ be a 5 or greater prime number, $z$ be an element of the parameters of elliptic curve $p$, and $P, Q$ be elements of $\mathrm{EC}_{\text {SetProjCo }}\left(z_{1}\right)$. Then $P \equiv Q$ if and only if $\left(\operatorname{compell}_{\mathrm{ProjCo}}(z, p)\right)(P) \equiv\left(\operatorname{compell}_{\mathrm{ProjCo}}(z, p)\right)(Q)$.

(47) Let $p$ be a 5 or greater prime number, $z$ be an element of the parameters of elliptic curve $p$, and $P, Q$ be elements of $\operatorname{EC}_{\text {SetProjCo }}\left(z_{1}\right)$. Then $P \equiv$ $\left(\operatorname{compell}_{\mathrm{ProjCo}}(z, p)\right)(Q)$ if and only if $\left(\operatorname{compell}_{\mathrm{ProjCo}}(z, p)\right)(P) \equiv Q$.

(48) Let $p$ be a 5 or greater prime number, $z$ be an element of the parameters of elliptic curve $p$, and $P, Q$ be elements of $\operatorname{EC}_{\text {SetProjCo }}\left(z_{1}\right)$. Suppose $P_{\mathbf{3}} \neq$ 0 and $Q_{\mathbf{3}} \neq 0$. Then the represent point of $P=\left(\operatorname{compell}_{\mathrm{ProjCo}}(z, p)\right)$ (the 
represent point of $Q)$ if and only if $P \equiv\left(\operatorname{compell}_{\text {ProjCo }}(z, p)\right)(Q)$.

(49) Let $p$ be a 5 or greater prime number, $z$ be an element of the parameters of elliptic curve $p$, and $P, Q$ be elements of $\operatorname{EC}_{\text {SetProjCo }}\left(z_{1}\right)$. If $P \equiv Q$, then $P_{2} \cdot Q_{3}=Q_{2} \cdot P_{3}$.

(50) Let $p$ be a 5 or greater prime number, $z$ be an element of the parameters of elliptic curve $p$, and $P, Q$ be elements of $\operatorname{EC}_{\text {SetProjCo }}\left(z_{\mathbf{1}}\right)$. Suppose $P_{\mathbf{3}} \neq$ 0 and $Q_{\mathbf{3}} \neq 0$. Then $P \equiv Q$ or $P \equiv\left(\operatorname{compell}_{\mathrm{ProjCo}}(z, p)\right)(Q)$ if and only if $P_{1} \cdot Q_{3}=Q_{1} \cdot P_{3}$.

(51) Let $p$ be a 5 or greater prime number, $z$ be an element of the parameters of elliptic curve $p$, and $P, Q$ be elements of $\operatorname{EC}_{\text {SetProjCo }}\left(z_{1}\right)$. If $P_{\mathbf{3}} \neq 0$ and $Q_{\mathbf{3}} \neq 0$ and $P_{\mathbf{2}} \neq 0$, then if $P \equiv\left(\operatorname{compell}_{\mathrm{ProjCo}}(z, p)\right)(Q)$, then $P_{2} \cdot Q_{3} \neq Q_{2} \cdot P_{3}$.

(52) Let $p$ be a 5 or greater prime number, $z$ be an element of the parameters of elliptic curve $p$, and $P, Q$ be elements of $\operatorname{EC}_{\text {SetProjCo }}\left(z_{1}\right)$. If $P \not \equiv Q$ and $P \equiv\left(\operatorname{compell}_{\mathrm{ProjCo}}(z, p)\right)(Q)$, then $P_{\mathbf{2}} \cdot Q_{\mathbf{3}} \neq Q_{\mathbf{2}} \cdot P_{\mathbf{3}}$.

(53) Let $p$ be a 5 or greater prime number, $z$ be an element of the parameters of elliptic curve $p, g_{5}$ be an element of $\operatorname{GF}(p)$, and $P$ be an element of $\mathrm{EC}_{\text {SetProjCo }}\left(z_{1}\right)$. If $g_{5}=3 \bmod p$ and $P_{\mathbf{2}}=0$ and $P_{\mathbf{3}} \neq 0$, then $z_{\mathbf{1}} \cdot\left(P_{\mathbf{3}}\right)^{2}+$ $g_{5} \cdot\left(P_{1}\right)^{2} \neq 0$.

(54) Let $p$ be a 5 or greater prime number, $z$ be an element of the parameters of elliptic curve $p, g_{4}, g_{6}, g_{7}, g_{8}$ be elements of $\mathrm{GF}(p), P, Q$ be elements of $\operatorname{EC}_{\text {SetProjCo }}\left(z_{1}\right)$, and $R$ be an element of (the carrier of $\left.\operatorname{GF}(p)\right) \times$ (the carrier of $\mathrm{GF}(p)) \times$ (the carrier of $\mathrm{GF}(p))$. Suppose that

(i) $g_{4}=2 \bmod p$,

(ii) $g_{6}=Q_{\mathbf{2}} \cdot P_{\mathbf{3}}-P_{\mathbf{2}} \cdot Q_{\mathbf{3}}$,

(iii) $g_{7}=Q_{1} \cdot P_{3}-P_{1} \cdot Q_{3}$,

(iv) $g_{8}=g_{6}{ }^{2} \cdot P_{\mathbf{3}} \cdot Q_{\mathbf{3}}-g_{7}{ }^{3}-g_{4} \cdot g_{7}{ }^{2} \cdot P_{\mathbf{1}} \cdot Q_{\mathbf{3}}$, and

(v) $\quad R=\left\langle g_{7} \cdot g_{8}, g_{6} \cdot\left(g_{7}^{2} \cdot P_{\mathbf{1}} \cdot Q_{\mathbf{3}}-g_{8}\right)-g_{7}{ }^{3} \cdot P_{\mathbf{2}} \cdot Q_{\mathbf{3}}, g_{7}{ }^{3} \cdot P_{\mathbf{3}} \cdot Q_{\mathbf{3}}\right\rangle$.

Then $g_{7} \cdot P_{\mathbf{3}} \cdot R_{\mathbf{2}}=-\left(g_{6} \cdot\left(R_{\mathbf{1}} \cdot P_{\mathbf{3}}-P_{\mathbf{1}} \cdot R_{\mathbf{3}}\right)+g_{7} \cdot P_{\mathbf{2}} \cdot R_{\mathbf{3}}\right)$.

(55) Let $p$ be a 5 or greater prime number, $z$ be an element of the parameters of elliptic curve $p, g_{4}, g_{6}, g_{7}, g_{8}$ be elements of GF $(p), P, Q$ be elements of $\operatorname{EC}_{\text {SetProjCo }}\left(z_{\mathbf{1}}\right)$, and $R$ be an element of (the carrier of $\left.\mathrm{GF}(p)\right) \times$ (the carrier of $\operatorname{GF}(p)) \times$ (the carrier of $\mathrm{GF}(p))$. Suppose that

(i) $g_{4}=2 \bmod p$,

(ii) $g_{6}=Q_{\mathbf{2}} \cdot P_{\mathbf{3}}-P_{\mathbf{2}} \cdot Q_{\mathbf{3}}$,

(iii) $g_{7}=Q_{1} \cdot P_{3}-P_{1} \cdot Q_{3}$,

(iv) $g_{8}=g_{6}{ }^{2} \cdot P_{\mathbf{3}} \cdot Q_{\mathbf{3}}-g_{7}{ }^{3}-g_{4} \cdot g_{7}^{2} \cdot P_{\mathbf{1}} \cdot Q_{\mathbf{3}}$, and

(v) $\quad R=\left\langle g_{7} \cdot g_{8}, g_{6} \cdot\left(g_{7}^{2} \cdot P_{\mathbf{1}} \cdot Q_{\mathbf{3}}-g_{8}\right)-g_{7}{ }^{3} \cdot P_{\mathbf{2}} \cdot Q_{\mathbf{3}}, g_{7}{ }^{3} \cdot P_{\mathbf{3}} \cdot Q_{\mathbf{3}}\right\rangle$.

Then $-g_{7}^{2} \cdot\left(P_{3} \cdot Q_{3} \cdot R_{1}+P_{3} \cdot Q_{1} \cdot R_{3}+P_{1} \cdot Q_{3} \cdot R_{3}\right)+P_{3} \cdot Q_{3} \cdot R_{3} \cdot g_{6}{ }^{2}=$ $0_{\mathrm{GF}(p)}$. 
(56) Let $p$ be a 5 or greater prime number, $z$ be an element of the parameters of elliptic curve $p, g_{4}, g_{6}, g_{7}, g_{8}$ be elements of $\mathrm{GF}(p), P, Q$ be elements of $\operatorname{EC}_{\text {SetProjCo }}\left(z_{1}\right)$, and $R$ be an element of (the carrier of $\left.\operatorname{GF}(p)\right) \times($ the carrier of $\operatorname{GF}(p)) \times$ (the carrier of $\operatorname{GF}(p))$. Suppose that

(i) $g_{4}=2 \bmod p$,

(ii) $g_{6}=Q_{\mathbf{2}} \cdot P_{\mathbf{3}}-P_{\mathbf{2}} \cdot Q_{\mathbf{3}}$,

(iii) $g_{7}=Q_{1} \cdot P_{3}-P_{1} \cdot Q_{3}$,

(iv) $g_{8}=g_{6}^{2} \cdot P_{\mathbf{3}} \cdot Q_{\mathbf{3}}-g_{7}{ }^{3}-g_{4} \cdot g_{7}^{2} \cdot P_{\mathbf{1}} \cdot Q_{\mathbf{3}}$, and

(v) $\quad R=\left\langle g_{7} \cdot g_{8}, g_{6} \cdot\left(g_{7}^{2} \cdot P_{\mathbf{1}} \cdot Q_{\mathbf{3}}-g_{8}\right)-g_{7}{ }^{3} \cdot P_{\mathbf{2}} \cdot Q_{\mathbf{3}}, g_{7}{ }^{3} \cdot P_{\mathbf{3}} \cdot Q_{\mathbf{3}}\right\rangle$.

Then $z_{\mathbf{2}} \cdot g_{7}^{2} \cdot\left(P_{\mathbf{3}}\right)^{2} \cdot Q_{\mathbf{3}} \cdot R_{\mathbf{3}}=-g_{7}^{2} \cdot P_{\mathbf{3}} \cdot P_{\mathbf{1}} \cdot Q_{\mathbf{1}} \cdot R_{\mathbf{1}}+\left(g_{7} \cdot P_{\mathbf{2}}-g_{6} \cdot P_{\mathbf{1}}\right)^{2}$. $Q_{3} \cdot R_{3}$.

(57) Let $p$ be a 5 or greater prime number, $z$ be an element of the parameters of elliptic curve $p, g_{4}, g_{6}, g_{7}, g_{8}$ be elements of $\mathrm{GF}(p), P, Q$ be elements of $\mathrm{EC}_{\mathrm{SetProjCo}}\left(z_{1}\right)$, and $R$ be an element of (the carrier of $\left.\mathrm{GF}(p)\right) \times($ the carrier of $\operatorname{GF}(p)) \times$ (the carrier of $\operatorname{GF}(p))$. Suppose that

(i) $g_{4}=2 \bmod p$,

(ii) $g_{6}=Q_{\mathbf{2}} \cdot P_{\mathbf{3}}-P_{\mathbf{2}} \cdot Q_{\mathbf{3}}$,

(iii) $g_{7}=Q_{1} \cdot P_{3}-P_{1} \cdot Q_{3}$,

(iv) $g_{8}=g_{6}^{2} \cdot P_{\mathbf{3}} \cdot Q_{\mathbf{3}}-g_{7}^{3}-g_{4} \cdot g_{7}^{2} \cdot P_{\mathbf{1}} \cdot Q_{\mathbf{3}}$, and

(v) $\quad R=\left\langle g_{7} \cdot g_{8}, g_{6} \cdot\left(g_{7}^{2} \cdot P_{\mathbf{1}} \cdot Q_{\mathbf{3}}-g_{8}\right)-g_{7}{ }^{3} \cdot P_{\mathbf{2}} \cdot Q_{\mathbf{3}}, g_{7}{ }^{3} \cdot P_{\mathbf{3}} \cdot Q_{\mathbf{3}}\right\rangle$.

Then $z_{\mathbf{1}} \cdot g_{7}^{2} \cdot P_{\mathbf{3}} \cdot Q_{\mathbf{3}} \cdot R_{\mathbf{3}}=g_{7}^{2} \cdot\left(P_{\mathbf{1}} \cdot Q_{\mathbf{1}} \cdot R_{\mathbf{3}}+P_{\mathbf{3}} \cdot Q_{\mathbf{1}} \cdot R_{1}+P_{\mathbf{1}} \cdot Q_{\mathbf{3}} \cdot\right.$ $\left.R_{\mathbf{1}}\right)+g_{4} \cdot g_{6} \cdot Q_{\mathbf{3}} \cdot R_{\mathbf{3}} \cdot\left(g_{7} \cdot P_{\mathbf{2}}-g_{6} \cdot P_{\mathbf{1}}\right)$.

(58) Let $p$ be a 5 or greater prime number, $z$ be an element of the parameters of elliptic curve $p, g_{4}, g_{6}, g_{7}, g_{8}$ be elements of $\mathrm{GF}(p), P, Q$ be elements of $\operatorname{EC}_{\text {SetProjCo }}\left(z_{1}\right)$, and $R$ be an element of (the carrier of $\left.\operatorname{GF}(p)\right) \times($ the carrier of $\operatorname{GF}(p)) \times$ (the carrier of $\operatorname{GF}(p))$. Suppose that

(i) $g_{4}=2 \bmod p$,

(ii) $g_{6}=Q_{\mathbf{2}} \cdot P_{\mathbf{3}}-P_{\mathbf{2}} \cdot Q_{\mathbf{3}}$,

(iii) $g_{7}=Q_{\mathbf{1}} \cdot P_{\mathbf{3}}-P_{\mathbf{1}} \cdot Q_{\mathbf{3}}$,

(iv) $g_{8}=g_{6}^{2} \cdot P_{\mathbf{3}} \cdot Q_{\mathbf{3}}-g_{7}^{3}-g_{4} \cdot g_{7}^{2} \cdot P_{\mathbf{1}} \cdot Q_{\mathbf{3}}$, and

(v) $\quad R=\left\langle g_{7} \cdot g_{8}, g_{6} \cdot\left(g_{7}^{2} \cdot P_{\mathbf{1}} \cdot Q_{\mathbf{3}}-g_{8}\right)-g_{7}{ }^{3} \cdot P_{\mathbf{2}} \cdot Q_{\mathbf{3}}, g_{7}{ }^{3} \cdot P_{\mathbf{3}} \cdot Q_{\mathbf{3}}\right\rangle$.

Then $g_{7}^{2} \cdot\left(P_{\mathbf{3}}\right)^{2} \cdot Q_{\mathbf{3}} \cdot\left(\left(R_{\mathbf{2}}\right)^{2} \cdot R_{\mathbf{3}}-\left(\left(R_{\mathbf{1}}\right)^{3}+z_{\mathbf{1}} \cdot R_{\mathbf{1}} \cdot\left(R_{\mathbf{3}}\right)^{2}+z_{\mathbf{2}} \cdot\left(R_{\mathbf{3}}\right)^{3}\right)\right)=0_{\mathrm{GF}(p)}$.

(59) Let $p$ be a 5 or greater prime number, $z$ be an element of the parameters of elliptic curve $p, g_{4}, g_{5}, g_{11}, g_{9}, g_{6}, g_{7}, g_{8}, g_{10}$ be elements of $\operatorname{GF}(p), P$ be an element of $\mathrm{EC}_{\mathrm{SetProjCo}}\left(z_{1}\right)$, and $R$ be an element of (the carrier of $\mathrm{GF}(p)) \times($ the carrier of $\mathrm{GF}(p)) \times$ (the carrier of $\mathrm{GF}(p)$ ). Suppose that $g_{4}=2 \bmod p$ and $g_{5}=3 \bmod p$ and $g_{11}=4 \bmod p$ and $g_{9}=8 \bmod p$ and $g_{6}=z_{\mathbf{1}} \cdot\left(P_{\mathbf{3}}\right)^{2}+g_{5} \cdot\left(P_{\mathbf{1}}\right)^{2}$ and $g_{7}=P_{\mathbf{2}} \cdot P_{\mathbf{3}}$ and $g_{8}=P_{\mathbf{1}} \cdot P_{\mathbf{2}} \cdot g_{7}$ and $g_{10}=g_{6}{ }^{2}-g_{9} \cdot g_{8}$ and $R=\left\langle g_{4} \cdot g_{10} \cdot g_{7}, g_{6} \cdot\left(g_{11} \cdot g_{8}-g_{10}\right)-g_{9} \cdot\left(P_{2}\right)^{2} \cdot g_{7}{ }^{2}\right.$, $\left.g_{9} \cdot g_{7}{ }^{3}\right\rangle$. Then $g_{4} \cdot g_{7} \cdot P_{\mathbf{3}} \cdot R_{\mathbf{2}}=-\left(g_{6} \cdot\left(P_{\mathbf{3}} \cdot R_{\mathbf{1}}-P_{\mathbf{1}} \cdot R_{\mathbf{3}}\right)+g_{4} \cdot g_{7} \cdot P_{\mathbf{2}} \cdot R_{\mathbf{3}}\right)$. 
(60) Let $p$ be a 5 or greater prime number, $z$ be an element of the parameters of elliptic curve $p, g_{4}, g_{5}, g_{11}, g_{9}, g_{6}, g_{7}, g_{8}, g_{10}$ be elements of GF $(p), P$ be an element of $\operatorname{EC}_{\text {SetProjCo }}\left(z_{1}\right)$, and $R$ be an element of (the carrier of $\mathrm{GF}(p)) \times($ the carrier of $\mathrm{GF}(p)) \times$ (the carrier of $\mathrm{GF}(p)$ ). Suppose that $g_{4}=2 \bmod p$ and $g_{5}=3 \bmod p$ and $g_{11}=4 \bmod p$ and $g_{9}=8 \bmod p$ and $g_{6}=z_{\mathbf{1}} \cdot\left(P_{\mathbf{3}}\right)^{2}+g_{5} \cdot\left(P_{\mathbf{1}}\right)^{2}$ and $g_{7}=P_{\mathbf{2}} \cdot P_{\mathbf{3}}$ and $g_{8}=P_{\mathbf{1}} \cdot P_{\mathbf{2}} \cdot g_{7}$ and $g_{10}=g_{6}{ }^{2}-g_{9} \cdot g_{8}$ and $R=\left\langle g_{4} \cdot g_{10} \cdot g_{7}, g_{6} \cdot\left(g_{11} \cdot g_{8}-g_{10}\right)-g_{9} \cdot\left(P_{2}\right)^{2} \cdot g_{7}^{2}\right.$, $\left.g_{9} \cdot g_{7}{ }^{3}\right\rangle$. Then $g_{11} \cdot g_{7}{ }^{2} \cdot P_{\mathbf{3}} \cdot R_{\mathbf{1}}=R_{\mathbf{3}} \cdot\left(g_{6}{ }^{2} \cdot P_{\mathbf{3}}-g_{9} \cdot g_{7}^{2} \cdot P_{\mathbf{1}}\right)$.

(61) Let $p$ be a 5 or greater prime number, $z$ be an element of the parameters of elliptic curve $p, g_{4}, g_{5}, g_{11}, g_{9}, g_{6}, g_{7}, g_{8}, g_{10}$ be elements of GF $(p), P$ be an element of $\operatorname{EC}_{\text {SetProjCo }}\left(z_{1}\right)$, and $R$ be an element of (the carrier of $\mathrm{GF}(p)) \times($ the carrier of $\mathrm{GF}(p)) \times$ (the carrier of $\mathrm{GF}(p))$. Suppose that $g_{4}=2 \bmod p$ and $g_{5}=3 \bmod p$ and $g_{11}=4 \bmod p$ and $g_{9}=8 \bmod p$ and $g_{6}=z_{\mathbf{1}} \cdot\left(P_{\mathbf{3}}\right)^{2}+g_{5} \cdot\left(P_{\mathbf{1}}\right)^{2}$ and $g_{7}=P_{\mathbf{2}} \cdot P_{\mathbf{3}}$ and $g_{8}=P_{\mathbf{1}} \cdot P_{\mathbf{2}} \cdot g_{7}$ and $g_{10}=$ $g_{6}{ }^{2}-g_{9} \cdot g_{8}$ and $R=\left\langle g_{4} \cdot g_{10} \cdot g_{7}, g_{6} \cdot\left(g_{11} \cdot g_{8}-g_{10}\right)-g_{9} \cdot\left(P_{2}\right)^{2} \cdot g_{7}{ }^{2}, g_{9} \cdot g_{7}{ }^{3}\right\rangle$. Then $g_{11} \cdot g_{7}^{2} \cdot\left(P_{\mathbf{3}}\right)^{2} \cdot\left(z_{\mathbf{2}} \cdot R_{\mathbf{3}}\right)=R_{\mathbf{3}} \cdot\left(g_{4} \cdot g_{7} \cdot P_{\mathbf{2}}-g_{6} \cdot P_{\mathbf{1}}\right)^{2}-g_{11} \cdot g_{7}^{2} \cdot\left(P_{\mathbf{1}}\right)^{2} \cdot R_{\mathbf{1}}$.

(62) Let $p$ be a 5 or greater prime number, $z$ be an element of the parameters of elliptic curve $p, g_{4}, g_{5}, g_{11}, g_{9}, g_{6}, g_{7}, g_{8}, g_{10}$ be elements of GF $(p), P$ be an element of $\operatorname{EC}_{\text {SetProjCo }}\left(z_{1}\right)$, and $R$ be an element of (the carrier of $\mathrm{GF}(p)) \times($ the carrier of $\mathrm{GF}(p)) \times$ (the carrier of $\mathrm{GF}(p)$ ). Suppose that $g_{4}=2 \bmod p$ and $g_{5}=3 \bmod p$ and $g_{11}=4 \bmod p$ and $g_{9}=8 \bmod p$ and $g_{6}=z_{\mathbf{1}} \cdot\left(P_{\mathbf{3}}\right)^{2}+g_{5} \cdot\left(P_{\mathbf{1}}\right)^{2}$ and $g_{7}=P_{\mathbf{2}} \cdot P_{\mathbf{3}}$ and $g_{8}=P_{\mathbf{1}} \cdot P_{\mathbf{2}} \cdot g_{7}$ and $g_{10}=g_{6}{ }^{2}-g_{9} \cdot g_{8}$ and $R=\left\langle g_{4} \cdot g_{10} \cdot g_{7}, g_{6} \cdot\left(g_{11} \cdot g_{8}-g_{10}\right)-g_{9} \cdot\left(P_{2}\right)^{2} \cdot g_{7}^{2}\right.$, $\left.g_{9} \cdot g_{7}{ }^{3}\right\rangle$. Then $g_{4} \cdot g_{7}{ }^{2} \cdot\left(P_{\mathbf{3}}\right)^{2} \cdot\left(z_{\mathbf{1}} \cdot R_{\mathbf{3}}\right)=g_{6} \cdot P_{\mathbf{3}} \cdot R_{\mathbf{3}} \cdot\left(g_{4} \cdot g_{7} \cdot P_{\mathbf{2}}-g_{6} \cdot\right.$ $\left.P_{\mathbf{1}}\right)+g_{7}^{2} \cdot\left(g_{11} \cdot P_{\mathbf{1}} \cdot P_{\mathbf{3}} \cdot R_{\mathbf{1}}+g_{4} \cdot\left(P_{\mathbf{1}}\right)^{2} \cdot R_{\mathbf{3}}\right)$.

(63) Let $p$ be a 5 or greater prime number, $z$ be an element of the parameters of elliptic curve $p, g_{4}, g_{5}, g_{11}, g_{9}, g_{6}, g_{7}, g_{8}, g_{10}$ be elements of $\mathrm{GF}(p), P$ be an element of $\operatorname{EC}_{\text {SetProjCo }}\left(z_{1}\right)$, and $R$ be an element of (the carrier of $\mathrm{GF}(p)) \times($ the carrier of $\mathrm{GF}(p)) \times$ (the carrier of $\mathrm{GF}(p)$ ). Suppose that $g_{4}=2 \bmod p$ and $g_{5}=3 \bmod p$ and $g_{11}=4 \bmod p$ and $g_{9}=8 \bmod p$ and $g_{6}=z_{\mathbf{1}} \cdot\left(P_{\mathbf{3}}\right)^{2}+g_{5} \cdot\left(P_{\mathbf{1}}\right)^{2}$ and $g_{7}=P_{\mathbf{2}} \cdot P_{\mathbf{3}}$ and $g_{8}=P_{\mathbf{1}} \cdot P_{\mathbf{2}} \cdot g_{7}$ and $g_{10}=$ $g_{6}{ }^{2}-g_{9} \cdot g_{8}$ and $R=\left\langle g_{4} \cdot g_{10} \cdot g_{7}, g_{6} \cdot\left(g_{11} \cdot g_{8}-g_{10}\right)-g_{9} \cdot\left(P_{2}\right)^{2} \cdot g_{7}{ }^{2}, g_{9} \cdot g_{7}{ }^{3}\right\rangle$. Then $g_{11} \cdot g_{7}^{2} \cdot\left(P_{\mathbf{3}}\right)^{2} \cdot\left(\left(R_{\mathbf{2}}\right)^{2} \cdot R_{\mathbf{3}}-\left(\left(R_{\mathbf{1}}\right)^{3}+z_{\mathbf{1}} \cdot R_{\mathbf{1}} \cdot\left(R_{\mathbf{3}}\right)^{2}+z_{\mathbf{2}} \cdot\left(R_{\mathbf{3}}\right)^{3}\right)\right)=0_{\mathrm{GF}}(p)$.

Let $p$ be a 5 or greater prime number and let $z$ be an element of the parameters of elliptic curve $p$. The functor $\operatorname{addell}_{\operatorname{ProjCo}}(z, p)$ yields a function from $\operatorname{EC}_{\text {SetProjCo }}\left(z_{1}\right) \times \operatorname{EC}_{\text {SetProjCo }}\left(z_{1}\right)$ into $\operatorname{EC}_{\text {SetProjCo }}\left(z_{1}\right)$ and is defined by the condition (Def. 9).

(Def. 9) Let $P, Q, O$ be elements of $\operatorname{EC}_{\text {SetProjCo }}\left(z_{1}\right)$ such that $O=\langle 0,1,0\rangle$. Then

(i) if $P \equiv O$, then $\left(\right.$ addell $\left._{\text {ProjCo }}(z, p)\right)(P, Q)=Q$,

(ii) if $Q \equiv O$ and $P \not \equiv O$, then $\left(\operatorname{addell}_{\mathrm{ProjCo}}(z, p)\right)(P, Q)=P$, 
(iii) if $P \not \equiv O$ and $Q \not \equiv O$ and $P \not \equiv Q$, then for all elements $g_{4}, g_{6}, g_{7}$, $g_{8}$ of $\mathrm{GF}(p)$ such that $g_{4}=2 \bmod p$ and $g_{6}=Q_{\mathbf{2}} \cdot P_{\mathbf{3}}-P_{\mathbf{2}} \cdot Q_{\mathbf{3}}$ and $g_{7}=Q_{\mathbf{1}} \cdot P_{\mathbf{3}}-P_{\mathbf{1}} \cdot Q_{\mathbf{3}}$ and $g_{8}=g_{6}{ }^{2} \cdot P_{\mathbf{3}} \cdot Q_{\mathbf{3}}-g_{7}{ }^{3}-g_{4} \cdot g_{7}{ }^{2} \cdot P_{\mathbf{1}} \cdot Q_{\mathbf{3}}$ holds $\left(\right.$ addell $\left._{\mathrm{ProjCo}}(z, p)\right)(P, Q)=\left\langle g_{7} \cdot g_{8}, g_{6} \cdot\left(g_{7}^{2} \cdot P_{\mathbf{1}} \cdot Q_{\mathbf{3}}-g_{8}\right)-g_{7}^{3} \cdot P_{\mathbf{2}} \cdot Q_{\mathbf{3}}\right.$, $\left.g_{7}^{3} \cdot P_{\mathbf{3}} \cdot Q_{\mathbf{3}}\right\rangle$, and

(iv) if $P \not \equiv O$ and $Q \not \equiv O$ and $P \equiv Q$, then for all elements $g_{4}, g_{5}, g_{11}$, $g_{9}, g_{6}, g_{7}, g_{8}, g_{10}$ of $\mathrm{GF}(p)$ such that $g_{4}=2 \bmod p$ and $g_{5}=3 \bmod p$ and $g_{11}=4 \bmod p$ and $g_{9}=8 \bmod p$ and $g_{6}=z_{\mathbf{1}} \cdot\left(P_{\mathbf{3}}\right)^{2}+g_{5} \cdot\left(P_{\mathbf{1}}\right)^{2}$ and $g_{7}=P_{\mathbf{2}} \cdot P_{\mathbf{3}}$ and $g_{8}=P_{\mathbf{1}} \cdot P_{\mathbf{2}} \cdot g_{7}$ and $g_{10}=g_{6}{ }^{2}-g_{9} \cdot g_{8}$ holds $\left(\right.$ addell $\left._{\text {ProjCo }}(z, p)\right)(P, Q)=\left\langle g_{4} \cdot g_{10} \cdot g_{7}, g_{6} \cdot\left(g_{11} \cdot g_{8}-g_{10}\right)-g_{9} \cdot\left(P_{2}\right)^{2} \cdot g_{7}^{2}\right.$, $\left.g_{9} \cdot g_{7}{ }^{3}\right\rangle$.

Let $p$ be a 5 or greater prime number, let $z$ be an element of the parameters of elliptic curve $p$, let $F$ be a function from $\operatorname{EC}_{\text {SetProjCo }}\left(z_{1}\right) \times \operatorname{EC}_{\text {SetProjCo }}\left(z_{1}\right)$ into $\mathrm{EC}_{\text {SetProjCo }}\left(z_{1}\right)$, and let $Q, R$ be elements of $\operatorname{EC}_{\text {SetProjCo }}\left(z_{1}\right)$. Then $F(Q, R)$ is an element of $\mathrm{EC}_{\mathrm{SetProjCo}}\left(z_{1}\right)$.

\section{REFERENCES}

[1] Grzegorz Bancerek. The ordinal numbers. Formalized Mathematics, 1(1):91-96, 1990.

[2] Czesław Byliński. Binary operations. Formalized Mathematics, 1(1):175-180, 1990.

[3] Czesław Byliński. Functions and their basic properties. Formalized Mathematics, 1(1):5565, 1990.

[4] Czesław Byliński. Functions from a set to a set. Formalized Mathematics, 1(1):153-164, 1990.

[5] Czesław Byliński. Some basic properties of sets. Formalized Mathematics, 1(1):47-53, 1990.

[6] Yuichi Futa, Hiroyuki Okazaki, and Yasunari Shidama. Set of points on elliptic curve in projective coordinates. Formalized Mathematics, 19(3):131-138, 2011, doi: 10.2478/v10037-011-0021-6.

[7] G. Seroussi I. Blake and N. Smart. Elliptic Curves in Cryptography. Number 265 in London Mathematical Society Lecture Note Series. Cambridge University Press, 1999.

[8] Eugeniusz Kusak, Wojciech Leończuk, and Michał Muzalewski. Abelian groups, fields and vector spaces. Formalized Mathematics, 1(2):335-342, 1990.

[9] Rafał Kwiatek. Factorial and Newton coefficients. Formalized Mathematics, 1(5):887-890, 1990.

[10] Rafał Kwiatek and Grzegorz Zwara. The divisibility of integers and integer relative primes. Formalized Mathematics, 1(5):829-832, 1990.

[11] Christoph Schwarzweller. The binomial theorem for algebraic structures. Formalized Mathematics, 9(3):559-564, 2001.

[12] Andrzej Trybulec. Domains and their Cartesian products. Formalized Mathematics, 1(1):115-122, 1990.

[13] Andrzej Trybulec. Tuples, projections and Cartesian products. Formalized Mathematics, 1(1):97-105, 1990.

[14] Michał J. Trybulec. Integers. Formalized Mathematics, 1(3):501-505, 1990.

[15] Wojciech A. Trybulec. Groups. Formalized Mathematics, 1(5):821-827, 1990.

[16] Wojciech A. Trybulec. Vectors in real linear space. Formalized Mathematics, 1(2):291-296, 1990.

[17] Zinaida Trybulec. Properties of subsets. Formalized Mathematics, 1(1):67-71, 1990.

Received November 3, 2011 